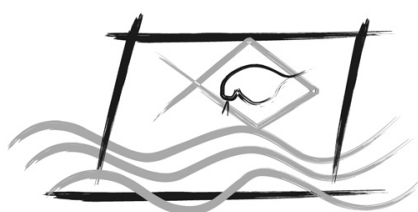

ECOTOX - BRASIL

\title{
Toxicidade e risco ambiental da oxitetraciclina e efeito em leucócitos de mato grosso (Hyphessobrycon eques)
}

\author{
R.Y.Fujimoto ${ }^{1}$; M.I. Gabbay ${ }^{2}$; E.C.S. Anjos ${ }^{2}$; S.P. Carraschi ${ }^{3}$ \& C. Cruz ${ }^{4}$ \\ ${ }^{1}$ Embrapa Tabuleiros Costeiros- Pesquisador, Av Beira Mar 3250 Caixa Postal 44 - CEP 49025-040 - Aracaju/SE - Brasil \\ ${ }^{2}$ Mestre em Ciência Animal pela Universidade Federal do Pará, Câmpus de Belém, Pará \\ ${ }^{3}$ Centro de Aquicultura da UNESP (CAUNESP), Câmpus de Jaboticabal, São Paulo \\ ${ }^{4}$ Doutor em Aquicultura e Pós-doutorando da Faculdade de Ciências Agrárias de Veterinárias da UNESP, Câmpus de Jaboticabal, São Paulo
}

(Received August 31, 2009; Accept August 18, 2010)

\begin{abstract}
Resumo
A oxitetraciclina (OTC) é um dos antibióticos mais utilizados para o tratamento de bacterioses de peixes. Os objetivos deste trabalho foram avaliar a toxicidade aguda e o risco ambiental e seus efeitos em leucócitos de mato grosso. Os peixes foram expostos a 2, $0 ; 4,0 ; 6,0$ e 8,0 $\mathrm{mg} \mathrm{L}^{-1}$ da OTC e um grupo utilizado como controle. Os testes foram conduzidos em um delineamento inteiramente casualizado, com quatro repetições e ao final do experimento, foram coletadas amostras de sangue para contagem dos leucócitos. Para a classificação do risco ambiental foi utilizada a $\mathrm{CL}_{50}$ e a concentração ambiental estimada (CAE) do antibiótico. A concentração letal 50\% (CL(I) 50 ;96h) estimada da OTC foi 5,49 $\mathrm{mg} \mathrm{L}^{-1}$ com limite inferior de 5,08 $\mathrm{mg} \mathrm{L}^{-1} \mathrm{e}$ superior de 5,95 $\mathrm{m} \mathrm{L}^{-1}$. A OTC causa moderado risco de intoxicação ambiental com a menor CAE e alto risco de intoxicação ambiental com a maior CAE. Não ocorreu diferença significativa na porcentagem de eosinófilos, célula granulocítica especial e basófilos, porém ocorreu linfocitopenia e monocitose em $4 \mathrm{mg} \mathrm{L}^{-1}$. Assim, a OTC é moderadamente tóxica e causa risco de intoxicação ambiental para o mato grosso e pode causar estresse em 4,0 e imunossupressão em $6,0 \mathrm{mg} \mathrm{L}^{-1}$.
\end{abstract}

Palavras-chaves: Peixe, Antibiótico, Concentração letal, Hematologia.

Toxicity and environmental risk of oxytetracycline and effect in leukocytes of mato grosso (Hyphessobrycon eques)

\begin{abstract}
The oxytetracycline (OTC) is one of antibiotic more used to control bacterial diseases on fishes. The objectives were to evaluate the toxicity of OTC and the environmental risk and its effects in leukocytes of Mato grosso (Hyphessobrycon eques). The fishes used in the experiment had average weight $0.22 \pm 0.2 \mathrm{~g}$ and they were acclimated for ten days in bioassay room and were exposed at $2.0 ; 4.0 ; 6.0$ and $8.0 \mathrm{mg} \mathrm{L}^{-1}$ of OTC and one treatment control. The tests were carried out in a completely randomized design, with four replicates and in the end, samples of blood were collected for leukocytes counting. For the classification of the environmental risk was utilized the $\mathrm{LC}_{50}$ and the estimated environmental concentration (CAE) of the antibiotic. The lethal concentration $50 \%$ (LC(I) 50 ;96h) estimated of the OTC was $5.49 \mathrm{mg} \mathrm{L}^{-1}$ with lower limit $5.08 \mathrm{mg} \mathrm{L}^{-1}$ and upper limit $5.95 \mathrm{mg} \mathrm{L}^{-1}$. The OTC cause moderately intoxication environmental risk with the small CAE and high intoxication environmental risk with the higher CAE. There were not differences $(\mathrm{p}>0.01)$ in percentages of eosinophils, granulocytic cells and basophils but occurred lymphopenia and monocytosis in $4.0 \mathrm{mg} \mathrm{L}^{-1}$. The OTC is moderately toxic and cause intoxication environmental risk for Mato grosso fish and caused stress in 4.0 and immune suppressor at $6.0 \mathrm{mg} \mathrm{L}^{-1}$
\end{abstract}

Keywords: Fish, Antibiotic, Lethal concentration, Hematology. 


\section{INTRODUÇÃO}

Um dos principais problemas da aquicultura moderna é a ocorrência de infecção dos peixes por parasitos e bactérias, principalmente em sistemas de criação intensivas (Cruz et al., 2004). As parasitoses e bacterioses destacam-se como importantes fatores limitantes da produtividade, pois provocam atraso no crescimento dos peixes e altas taxas de mortalidade (Ranzani-Paiva et al., 1997).

Entre os antibióticos, a oxitetraciclina (OTC) é um dos mais utilizados para tratar infecções causadas por uma grande variedade de bactérias (Rigos \& Troisi, 2005). A OTC é o ingrediente ativo da formulação Terramicina ${ }^{\circledR}$, um bacteriostático que atua sobre bactérias Gram positivas e Gram negativas, inibindo a síntese protéica (Rigos et al., 2006).

Os impactos e efeitos adversos provocados por diversas substâncias químicas sobre a biota aquática podem ser avaliados e mensurados por estudos ecotoxicológicos que permitem avaliar a sensibilidade de organismos aquáticos a diversas fontes poluidoras (Lombardi, 2004).

Dentre as análises utilizadas para avaliação dos possíveis efeitos ambientais como as alterações na qualidade da água por meio da presença de xenobióticos, manejo inadequado e altas densidades de estocagem, que podem alterar o sistema imune dos organismos aquáticos, a análise sanguínea se destaca. O sangue é um tecido alvo muito utilizado em testes de toxicidade para determinar efeitos deletérios de substâncias químicas (Ferreira, 2004).

Além disso, a avaliação de risco ambiental é outra ferramenta muito importante de análise do impacto dos compostos tóxicos e correlaciona a toxicidade aguda, em determinadas condições de laboratório, com a concentração ambiental estimada (CAE) da recomendação de uso (Zagatto $\&$ Bertoletti, 2008).

Poucos estudos são realizados para avaliar a toxicidade aguda de antibióticos, especialmente para peixes tropicais. Além disso, estudos sobre as repostas hematológicas a esses farmoquímicos são importantes para avaliar a possibilidade de utilização de antibióticos. Assim, os objetivos deste trabalho foram: avaliar a toxicidade aguda CL (I) $)_{50} ; 96 \mathrm{~h}$ do antibiótico OTC na formulação Terramicina ${ }^{\circledR}$ em jovens de mato grosso e os possíveis efeitos sobre os leucócitos.

\section{MATERIAL E MÉTODOS}

O experimento foi realizado no Laboratório de Crustáceos da Universidade Federal do Pará, Campus de Bragança. Os animais foram mantidos durante 10 dias em caixas de polietileno com capacidade para $300 \mathrm{~L}$. Os peixes foram alimentados duas vezes ao dia até a saciedade com ração comercial e renovação de água constante.

\section{Teste de sensibilidade e testes preliminares de toxicidade aguda}

Os peixes com peso médio de $0,22 \pm 0,2 \mathrm{~g}$ foram previamente aclimatados por dez dias em sala de bioensaio e alimentados até a saciedade com ração comercial.

Para o controle de sensibilidade e sanidade dos organismos teste foi utilizado o cloreto de potássio $(\mathrm{KCl})$ com teor de pureza de $99 \%$, como substância referência. A concentração letal $50 \%\left(\mathrm{CL}(\mathrm{I})_{50} ; 96 \mathrm{~h}\right)$ dessa substância para o mato grosso foi de $1,5 \mathrm{~g} \mathrm{~L}^{-1}$.

Após a avaliação da sensibilidade dos organismos teste foram realizados testes preliminares com a OTC para determinar as concentrações que causam zero e $100 \%$ de mortalidade.

\section{Teste definitivo de toxicidade aguda}

Nos testes definitivos foram utilizadas quatro concentrações de OTC $\left(2,0 ; 4,0 ; 6,0\right.$; e $\left.8,0 \mathrm{mg} \mathrm{L}^{-1}\right)$ e um controle, com cinco peixes por tratamento, com quatro repetições por concentração.

Os testes foram conduzidos em sistema estático, em delineamento inteiramente casualizado (DIC) com duração de 96 horas. A mortalidade foi avaliada a cada seis horas com a retirada dos peixes mortos. Diariamente também foram monitoradas as variáveis de qualidade de água $(\mathrm{pH}$, oxigênio dissolvido e temperatura).

A estimativa da concentração letal $\left(\mathrm{CL}(\mathrm{I})_{50} ; 96 \mathrm{~h}\right)$ foi realizada pela metodologia de Trimmed Spearman Karber (Hamilton et al., 1977).

Após a obtenção desses valores de $\mathrm{CL}_{50}$, a OTC foi classificada quanto à toxicidade aguda de acordo com as classes de toxicidade descritas por Zucker (1985).

\section{Risco Ambiental}

A OTC foi classificada nas classes de quociente de risco ambiental calculados pela razão entre a concentração ambiental estimada (CAE) e o valor da $\mathrm{CL}(\mathrm{I})_{50} ; 48 \mathrm{~h}$. As classes de risco ambiental utilizadas foram: sem risco $(\mathrm{Q}<0,1)$; risco baixo $(0,1<\mathrm{Q}<10)$; risco moderado $(1<\mathrm{Q}<10)$ e risco alto $(\mathrm{Q}>10)$ (USEPA, 1987). O risco de intoxicação ambiental da OTC foi estimado para a menor dose recomendada $(50,0 \mathrm{mg} \mathrm{kg}$ 1, Ueno, et al., 2003) e para a maior $\left(1.750,0 \mathrm{mg} \mathrm{kg}^{-1}\right.$, FDA, $1998)$ e a $\mathrm{CL}(\mathrm{I})_{50} ; 48 \mathrm{~h}$ para o mato grosso.

\section{Contagem diferencial de leucócitos}

Ao término do teste definitivo foram coletadas amostras de sangue de quatro peixes de cada tratamento.

Devido ao tamanho pequeno dos peixes, estes foram anestesiados (benzocaina $0,05 \mathrm{mg} \mathrm{L}^{-1}$ ), o sangue foi retirado por corte da nadadeira caudal. Uma gota foi utilizada para a confecção da extensão sanguínea, que foi seca à temperatura ambiente e posteriormente corada com Leishman para a contagem diferencial de leucócitos. Após a obtenção dos dados, estes foram submetidos aos testes de premissa de normalidade de Shpairo-Wilk para então ser realizada a Analise 
de Variância $(\mathrm{p}=0,01)$, quando o $\mathrm{F}$ foi significativo foi realizado o teste de Tukey $(\mathrm{p}=0,05)$ para comparação de médias.

\section{RESULTADOS E DISCUSSÃO}

Durante a realização do teste de toxicidade aguda o $\mathrm{pH}$ variou de 6,9 a 7,4; permanecendo próximo a neutralidade, a temperatura de 26,3 a $27,9{ }^{\circ} \mathrm{C}$ e o oxigênio dissolvido variou de 4,7 a 7,4 $\mathrm{mg} \mathrm{L}^{-1}$, porém, nenhuma destas variáveis foi restritiva para a manutenção dos peixes nesta condição, permanecendo dentro dos valores recomendados pela ABNT (2004).

A concentração letal $50 \%\left(\mathrm{CL}(\mathrm{I})_{50} ; 96 \mathrm{~h}\right)$ estimada da OTC para o mato grosso foi de $5,49 \mathrm{mg} \mathrm{L}^{-1}$ com limite superior de 5,95 $\mathrm{mg} \mathrm{L}^{-1} \mathrm{e}$ inferior de 5,08 $\mathrm{mg} \mathrm{L}^{-1}$.

$\mathrm{Na}$ concentração de $2,0 \mathrm{mg} \mathrm{L}^{-1}$ a taxa de mortalidade foi de 5\%; em 4,0 $\mathrm{mg} \mathrm{L}^{-1}$, não ocorreu mortalidade; em $6,0 \mathrm{mg} \mathrm{L}^{-1}$ foi de $65 \%$, e em $8,0 \mathrm{mg} \mathrm{L}^{-1}, 100 \%$. Porém, nas primeiras seis horas de exposição, ocorreu mortalidade de $95 \%$ dos peixes em $8,0 \mathrm{mg} \mathrm{L}^{-1}$.

Segundo a classificação de Zucker (1985), a OTC é considerada moderadamente tóxica para o mato grosso, pois apresentou $\mathrm{CL}_{50}$ entre 1,0 e $10,0 \mathrm{mg} \mathrm{L}^{-1}$.

A OTC foi menos tóxica para o mato grosso do que para a alga Pseudokirchneriella subcapitata $\left(\mathrm{CE}_{50} ; 72 \mathrm{~h} 0,17 \mathrm{mg}\right.$ $\mathrm{L}^{-1}$; Nunes et al., 2005) e para macrófita Lemna gibba com $\mathrm{CE}_{50} ; 7 \mathrm{~d}$ de $1,15 \mathrm{mg} \mathrm{L}^{-1}$ (Brain et al., 2004).

Quando comparada a outros produtos utilizados para o tratamento de bacterioses, ectoparasitos e algas, a OTC foi menos tóxica para o mato grosso que o permanganato de potássio para Morone saxatilus (CL(I) $; 0$; $96 \mathrm{~h}$ de 0,96 mg L-1; Reardon \& Harrell, 1994) e que o sulfato cobre para a carpa (Labeo rohita) (CL (I) 50 ; $96 \mathrm{~h} \mathrm{de} 0,56 \mathrm{mg} \mathrm{L}^{-1}$; Adhikari, 2003).

A OTC foi mais tóxica para o mato grosso do que para $S$. namaycush $\left(\mathrm{CL}_{50} ; 24 \mathrm{~h}>200 \mathrm{mg} \mathrm{L}^{-1}\right.$; Webb, 2001), $M$. saxatilis $\left(\mathrm{CL}_{50} ; 48 \mathrm{~h}\right.$ de $125,0 \mathrm{mg} \mathrm{L}^{-1}$; Boxall et al., 2002), D. rerio $\left(\mathrm{CL}_{50} ; 96 \mathrm{~h}>1000 \mathrm{mg} \mathrm{L}^{-1}\right.$; Isidori et al., 2005), O. latipes $\left(\mathrm{CL}_{50} ; 48 \mathrm{~h}\right.$ de $\left.110,1 \mathrm{mg} \mathrm{L}{ }^{-1} ; \mathrm{Kim}, 2007\right)$ e que o formaldeído para a tilápia do Nilo (Oreochromis niloticus) (CL (I) $)_{50}$;96h de 429,68 $\mathrm{mg} \mathrm{L}^{-1}$; Macniven \& Little, 2001).

A OTC apresenta moderado risco de intoxicação ambiental $(\mathrm{Q}=9,1)$ quando utilizando a menor CAE encontrada na literatura $\left(\mathrm{CAE}=50,0 \mathrm{mg} \mathrm{kg}^{-1}\right)$, pois $1<\mathrm{Q}$ $<10$, porém é de alto risco de intoxicação ambiental $(\mathrm{Q}=$ $318,76)$ quando utilizando a maior $\mathrm{CAE}(\mathrm{CAE}=1750,0 \mathrm{mg}$ $\mathrm{kg}^{-1}$ ), pois $\mathrm{Q}>10$.

$\mathrm{O}$ risco de intoxicação ambiental do mato grosso pelo uso da OTC na maior CAE é maior que a dos antibióticos mecilinam e trimetropina para $D$. rerio $(\mathrm{Q}<1)$ (HallingSorensen et al., 2000). Na menor CAE o risco de intoxicação ambiental do mato grosso pela OTC é semelhante ao risco para O. latipes (Boxall et al. 2002; Park \& Choi, 2008) e da ciprofloxacina para D. rerio (Halling-Sorensen et al., 2000).

Em relação ao sangue, não ocorreu diferença significativa na porcentagem de eosinófilos, célula granulocítica especial e basófilos nas concentrações avaliadas, porém ocorreu linfocitopenia e monocitose na concentração de $4,0 \mathrm{mg} \mathrm{L}^{-1}$ (Fig. 1A e 1C).

Os neutrófilos apresentaram um aumento nas concentrações de 4,0 e 6,0 $\mathrm{mg} \mathrm{L}^{-1}$, porém não apresentaram diferenças significativas. Assim, ocorreu uma linfocitopenia e uma neutrofilia aparente em 4,0 e $6,0 \mathrm{mg} \mathrm{L}^{-1}$ caracterizando uma resposta a um agente estressor. Segundo Mazeuaud et al. (1977), um agente estressor pode desencadear a síndrome geral de adaptação. Essa síndrome descrita primeiramente por Selye (1950) é caracterizada por três fases: a de alarme, adaptação e exaustão. E uma linfocitopenia e a neutrofilia caracterizam um reflexo da resposta terciária ao estresse (Mazeuaud et al., 1977).

$\mathrm{Na}$ exposição à OTC ocorreu linfocitopenia na concentração de 4,0 mg L-1 (Fig. 1A). Lundem et al. (1998) também apontaram a redução de leucócitos circulantes, principalmente os linfócitos (linfocitopenia) durante o tratamento com OTC em truta arco-íris (Oncorhynchus mykiss), indicando supressão do sistema imunológico.

Também ocorreu um aumento dos monócitos (fig. 1C) e um possível aumento dos neutrófilos ( $>00,05)$ em $4,0 \mathrm{mg}$ $\mathrm{L}^{-1}$ e uma diminuição em $6,0 \mathrm{mg} \mathrm{L}^{-1}$ (Fig. 1B). Esse aumento de células fagocitárias também ocorreu em Sparus auratus, durante o tratamento com OTC na dieta com concentração de 50 a $75 \mathrm{mg} \mathrm{kg}^{-1}$ de peso vivo (Serezli et al., 2005). Após os dez dias de tratamento ocorreu uma supressão do sistema imune devido a ação mais prolongada do xenobiótico, o que também pode estar relacionada com a maior concentração de OTC $\left(6,0 \mathrm{mg} \mathrm{L}^{-1}\right)$ neste trabalho.

Embora sem diferença significativa, (devido ao alto coeficiente de variação) ocorreu um possível aumento nos granulócitos neutrófilos, eosinófilos, CGE e basófilos (Fig. 1B, 1D, 1E e 1F) diferindo de Lundem et al. (1998) que ressalta uma diminuição dos granulócitos em Oncorhynchus mykiss.

Segundo Ferreira (2004) as alterações sanguíneas mais importantes após um teste de toxicidade aguda nos organismos alvos são metahemoglobinemias, anemias hemolíticas, citopenias e alterações nas quantidades de leucócitos. No presente trabalho observou-se alterações nos leucócitos, principalmente linfocitopenia e monocitose, em 4,0 e 6,0 $\mathrm{mg} \mathrm{L}^{-1}$.

Assim pode-se concluir que a OTC na formulação Terramicina $^{\circledR}$ é moderadamente tóxica e causa risco de intoxicação ambiental para jovens de mato grosso $(H$. eques), apresentando $\mathrm{CL}_{50}$ de $5,49 \mathrm{mg} \mathrm{L}^{-1}$. A OTC causa estresse em exposição a $4 \mathrm{mg} \mathrm{L}^{-1}$ e imunossupressão a 6 $\mathrm{mg} \mathrm{L}{ }^{-1}$, sugerindo que $4 \mathrm{mg} \mathrm{L}^{-1}$ pode ser um limiar entre o benefício do antibiótico e uma possível ação negativa como a imunossupressão. 

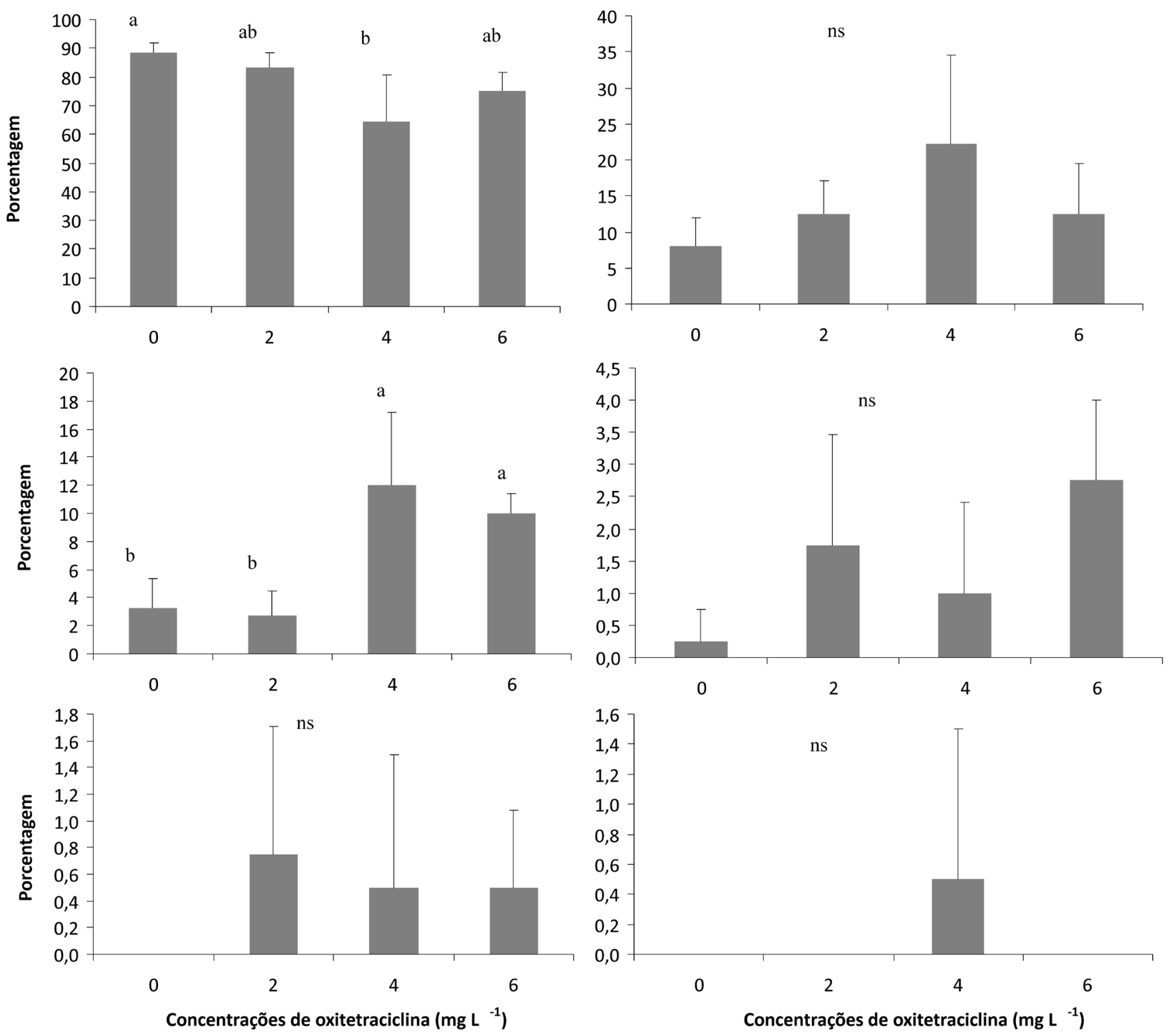

Figura 1. Valores percentuais médios e desvio padrão de: A- linfócitos, B- neutrófilos, C- monócitos, D- eosinófilos, E- célula granulocítica especial (CGE) e F-basófilo de mato grosso expostos a OTC. ns: não significativo. Médias com letras semelhantes em cada figura não apresentam diferenças significativas pelo teste de Tukey.

\section{REFERÊNCIAS}

ABNT. Associação Brasileira de Normas Técnicas, 2004, NBR 15088: Ecotoxicologia aquática - Toxicidade aguda - Método de ensaio com peixes. São Paulo, 19p.

ADHIKARI, S., 2003, Effect of calcium and magnesium hardness on acute copper toxicity to Indian major carp, Labeo rohita (Hamilton) and catfish, Channa punctatus (Bloch). Aquacul. Res., Oxford, 34: 975-980. DOI: 10.1046/j.1365-2109.2003.00895.x

BRAIN R.A, JOHNSON D.J, RICHARDS S.M, SANDERSON H, SIBLEY P.K, \& SOLOMON K.R, 2004, Effects of 25 pharmaceutical compounds to Lemna gibba using a seven-day static-renewal test. Environ. Toxicol. Chem., 23:371-382. DOI: 10.1897/02-576

BOXALL, A. B. A.; FOGG, L.; BLACKWELL, P. A.; KAY, P. \& PEMBERTON, E., 2002, Review of Veterinary Medicines in the Environment. Environ. Ag. R\&D Tech. Rep., P6-002/TR, ISBN 1-8570-5-780-5.
CRUZ, C., MACHADO-NETO, J. G. \& MENEZES, M. L., 2004, Toxicidade aguda do inseticida paration metílico e do biopesticida azadiractina de folhas de neem (Azadirachta indica) para alevino e juvenil de pacu (Piaractus mesopotamicus). Pesticidas: $R$. Ecotoxicol. Meio Ambien, 14: 93-102.

FDA, Food and Drug Administration. 1998. "Chapter 11: Aquaculture Drugs." In Fish and Fishery Products Hazards and Controls Guide. (Second Edition). FDA: Washington, D.C., 115-132.

FERREIRA, C. M., 2004, Análises complementares obtidas a partir de testes de toxicidade aquática. In: Sanidade De Organismos Aquáticos. Ranzani Paiva, M. J. T., Takemoto R. M., Lizama, M. A. P., Ed. Varela, São Paulo, 273-284.

HALLING-SǾRENSEN, B., HOLTEN LUTZHǾFT, H. C., ANDERSEN, H. R. \& INGERSLEV, F., 2000, Environmental risk assessment of antibiotics: comparison of mecillinam, trimetropim and ciprofloxacin. Journal Antimicrobial Chemotherapy, Supplied. S1, v. 46, p. 53-58.. doi: 10.1093/ jac/46.suppl_1.53 
HAMILTON, M. A., RUSSO, R. C. \& THURSTON, V., 1977, Trimmed Spearman-Karber method for estimating medial lethal concentrations in toxicity bioassays. Environ. Scien. Techonol., Iowa, 7: 714-719. doi: 10.1021/es60130a004

ISIDORI, M.; LAVORGNA, M.; NARDELLI, A.; PASCARELLA, L. \& PARELLA, A., 2005, Toxic and genotoxic evaluation of six antibiotics on non-target organisms. Sc. Tot. Environ., 346: 87-98. doi:10.1016/j.scitotenv.2004.11.017

KIM, Y.; CHOI, K.; JUNG, J.; PARK, S.; KIM, P. \& PARK, J., 2007, Aquatic toxicity of cetaminophen, carbamazepine, cimetidine, diltiazem and six major sulfonamides, and their potential ecological risks in Korea. Environ. Intern., 33: 370375. doi:10.1016/j.envint.2006.11.017

LOMBARDI, J. V., 2004, Fundamentos de toxicologia aquática In: Sanidade De Organismos Aquáticos. RANZANI PAIVA, M. J. T., TAKEMOTO, R. M., LIZAMA, M. A. P., Ed. Varela, São Paulo, 263-272.

LUNDEM, T., MIETTINEN, S., LÖNNSTRÖM, L. G., LILIUS, E. M. \& BYLUND, G., 1998, Influence of oxytetracycline and oxolinic acid on the immune response of rainbow trout (Oncorhynchus mykiss). Fish \& Shell. Immunol., Aberdeen, 8: 217-230.

MACNIVEN, A. M. \& LiTTLE, D. C., 2001, Development and evaluation of a stress challenge testing methodology for assessment of Nile tilapia (Oreochromis niloticus, Linn.) fry quality. Aquacul. Res., Oxford, 32: 671-679. doi: 10.1046/j.13652109.2001.00574.x

MAZEAUD, M.M.; MAZEAUD, F. \& DONALDSON, E.M. 1977. Primary and secondary effects of stress in fish: some new data with a general review. Transac. Am. Fish. Soc., 106, 201-212. doi:10.1577/1548-8659(1977)106<201:PASEOS >2.0.CO;2

NUNES, B.; CARVALHO, F. \& GUILHERMINO, L. Acute toxicity of widely used pharmaceuticals in aquatic species, Gambusia holbrooki, Artemia parthenogenetica and Tetraselmi chuii. Ecotox. Environ. Saf., v. 61, p 413-419, 2005. doi:10.1016/j. ecoenv.2004.08.010

PARK, S. \& CHOI, K., 2008, Hazard assessment of commonly used agricultural antibiotics on aquatic ecosystems, Ecotoxicology, v. 17, p. 526-538.. doi: 10.1007/s10646-008-0209-x

RANZANI-PAIVA, M. J. T., ISHIKAWA, C. M., CAMPOS, B. E. S. \& EIRAS, A. C., 1997, Hematological characteristics associated with parasitism in mullets, Mugil platanus, from the estuarine region of Cananéia, São Paulo, Brasil. Rev. Bras. Zool., São Paulo, 14(2): 329-339.

REARDON, I. S.\& HARRELL, R. M., 1994, Effects of varying salinities on the toxicity of potassium permanganate to larval and juvenile striped bass, Morone saxatilis (Walbaum). Aquacul. Res., Oxford, 25: 571-578. doi: 10.1111/j.1365-2109.1994. tb00721.x

RIGOS, G. \& TROISI, G. M., 2005, Antibacterial agents in Maditerranean finfish farming: A sinopsis of drug pharmacokinetics in important euryhaline fish species and possible environmental implications. Rev. Fish Biol. Fisher., 15: 53-73. doi 10.1007/s11160-005-7850-8

RIGOS, G., NENGAS, I. \& ALEXIS, M., 2006, Oxytetracycline (OTC) uptake following bath treatment in gilthead sea bream (Sparus aurata). Aquac., New York, 261: 1151-1155. doi:10.1016/j.aquaculture.2006.09.023

SELYE, H. 1950 Stress and general adaptation syndrome. British Medical Journal, 1(4667): 1383-1392.

SEREZLI, R., AÚIRGAN, H., OKUMUS, Ü., AKHAN, S. L. \& BALTA, F., 2005, The effect of oxytetracycline on non-specific immune response in sea bream (Sparus aurata L. 1758). Turk. $J$. Vet. Anim. Sci., Istambul, 29: 31-35.

UENO, R. KINOSHITA, A. \& WAKABAYASHI, J. 2003. Comparative pharmacokinetics of oxytetracycline in eel and its fate in a closed aquatic environment. Aquaculture, 235, 53-63.

USEPA, 1987. Assessment of health risks to garment workers and certain home residents from exposure to formaldehyde. Office of Pesticides and Toxic Substances, Environmental Protection Agency, Washington DC.

WEBB, S. F., 2001, A data-based perspective on the environmental risk assessment of human pharmaceuticals. I. Collation of vailable ecotoxicity data. In: Kümmerer K (ed) Pharmaceuticals in the environment: sources, fate, effects and risks. Springer, Heidelberg, 175-201.

ZAGATTO, P. A., \& E. BERTOLETTI, 2008, Ecotoxicologia Aquática - Princípios e Aplicações, Editora Rima, São Carlos, $478 \mathrm{p}$.

ZUCKER, E., 1985, Hazard Evaluation Division - Standard Evaluation Procedure - Acute Toxicity Test for Freshwater Fish. USEPA Publication, Washington, 540:85-006. 